\title{
Microtiter plate cultivation of oleaginous fungi and monitoring of lipogenesis by high-throughput FTIR spectroscopy
}

Gergely Kosa ${ }^{1 *} \mathbb{C}$, Achim Kohler ${ }^{1}$, Valeria Tafintseva ${ }^{1}$, Boris Zimmermann ${ }^{1}$, Kristin Forfang ${ }^{1}$, Nils Kristian Afseth ${ }^{2}$, Dimitrios Tzimorotas ${ }^{2}$, Kiira S. Vuoristo ${ }^{3}$, Svein Jarle Horn³ ${ }^{3}$ Jerome Mounier ${ }^{4}$ and Volha Shapaval ${ }^{1}$

\begin{abstract}
Background: Oleaginous fungi can accumulate lipids by utilizing a wide range of waste substrates. They are an important source for the industrial production of omega- 6 polyunsaturated fatty acids (gamma-linolenic and arachidonic acid) and have been suggested as an alternative route for biodiesel production. Initial research steps for various applications include the screening of fungi in order to find efficient fungal producers with desired fatty acid composition. Traditional cultivation methods (shake flask) and lipid analysis (extraction-gas chromatography) are not applicable for large-scale screening due to their low throughput and time-consuming analysis. Here we present a microcultivation system combined with high-throughput Fourier transform infrared (FTIR) spectroscopy for efficient screening of oleaginous fungi.
\end{abstract}

Results: The microcultivation system enables highly reproducible fungal fermentations throughout 12 days of cultivation. Reproducibility was validated by FTIR and HPLC data. Analysis of FTIR spectral ester carbonyl peaks of fungal biomass offered a reliable high-throughput at-line method to monitor lipid accumulation. Partial least square regression between gas chromatography fatty acid data and corresponding FTIR spectral data was used to set up calibration models for the prediction of saturated fatty acids, monounsaturated fatty acids, polyunsaturated fatty acids, unsaturation index, total lipid content and main individual fatty acids. High coefficients of determination $\left(\mathrm{R}^{2}=0.86-\right.$ $0.96)$ and satisfactory residual predictive deviation of cross-validation $\left(\mathrm{RPD}_{\mathrm{CV}}=2.6-5.1\right)$ values demonstrated the goodness of these models.

Conclusions: We have demonstrated in this study, that the presented microcultivation system combined with rapid, high-throughput FTIR spectroscopy is a suitable screening platform for oleaginous fungi. Sample preparation for FTIR measurements can be automated to further increase throughput of the system.

Keywords: Microcultivation, Oleaginous fungi, Fatty acid analysis, GC-FID, High-throughput FTIR spectroscopy, PLS regression

\section{Background}

Generally, a microorganism is considered oleaginous if the lipid content exceeds $20 \%$ of its dry weight, while up to $70 \%$ lipid content has been reported in the literature [1]. To achieve such high lipid content, microorganisms need to be cultivated in excess of a carbon source, while

\footnotetext{
*Correspondence: gergely.kosa@nmbu.no

1 Faculty of Science and Technology, Norwegian University of Life Sciences, Postbox 5003, 1432 Ås, Norway

Full list of author information is available at the end of the article
}

other nutrients such as nitrogen should be present in limiting concentration, i.e. in a high carbon-to-nitrogen ratio. Oleaginous microorganisms respond to nitrogen depletion by accumulating carbon in the form of triacylglycerol (TAG) in distinct lipid bodies. Oleaginous species can be found among yeasts, filamentous fungi and microalgae, while bacteria usually produce polyhydroxybutyrate and polyhydroxyalkanoate as storage polymers [1].

Microbial oils or single cell oils (SCO) are important sources of high-value polyunsaturated fatty acids (PUFA) 
for human consumption as nutraceuticals. Plants do not produce PUFA longer than C18 (trienoic acids), while fish oil has several disadvantages, such as odor, accumulated toxic compounds, and overfishing $[1,2]$. The commercially produced microbial oils contain high percentage of polyunsaturated fatty acids (PUFA). For example, omega-6 PUFAs, such as gamma-linolenic acid (18:3, GLA) and arachidonic acid (20:4, ARA) have been produced by the filamentous fungi Mucor circinelloides and Mortierella alpina, respectively.

Initial steps in microbial lipid research involve the screening for efficient production strains, which are either genetically modified organisms or natural isolates, and media optimization for subsequent scale-up experiments. When a large number of strains or cultivation conditions have to be tested, a high throughput screening (HTS) system is required, which can yield reproducible and scalable results. In order to increase throughput compared to traditional shake flasks, miniaturization of cultivations by microtiter plates or microbioreactors is desired [3-5]. HTS of filamentous fungi in microtiter plates is a challenging task because (a) there is a substantial risk of cross-contamination between individual cultivations, (b) highly viscous fermentation broth can cause oxygen transfer limitation and local inhomogeneity, and (c) excessive wall growth may take place, which favors sporulation.

For the screening of oleaginous microorganisms in microplates, a rapid, accurate lipid analysis is desired. Extraction, transesterification and gas chromatography (GC) is time-consuming, expensive and requires sample preparation and analysis that creates toxic wastes [6-8]. Rapid, non-invasive methods for SCO analysis involve fluorescence based measurements, near-infrared spectroscopy (NIR), Fourier transformed infrared spectroscopy (FTIR) and Raman spectroscopy [9]. FTIR spectroscopy has been successfully applied in recent years for microbial lipid research in yeast $[8,10,11]$, microalgae [7, 12-14] and filamentous fungi [15]. FTIR spectroscopy is extremely versatile since it enables the overall characterization of the biochemical composition of intact cells, including proteins, lipids, and carbohydrates [11]. For that reason, FTIR spectroscopy is widely used for the rapid differentiation and identification of microorganisms [16-19]. The main advantages of FTIR spectroscopy are that (a) several compounds can be measured simultaneously, (b) the method is rapid since little or no sample preparation is required for spectral acquisition, (c) it is chemical-free, (d) it can be used for HTS and for real-time bioprocess monitoring, (e) and even spatial information can be obtained by the use of FTIR microspectroscopy systems [20,21]. Since infrared spectra are highly complex, with many overlapping signals, multivariate data analysis is required to gain useful information $[17,22]$.

The aim of this study was to introduce the Duetz microtiter plate system (Duetz-MTPS) combined with HTS-FTIR spectroscopy as a high-throughput analytical platform for screening and monitoring of oleaginous fungi. In order to demonstrate the suitability of the system, we have used three fungal species and incubated them for 12 days under different temperatures. We have monitored lipogenesis of the fungal fermentations in microplates by high-throughput FTIR spectroscopy and $\mathrm{GC}$ reference analysis.

\section{Methods}

\section{Fungal strains}

Three oleaginous filamentous fungi were used in this study: Mucor circinelloides VI 04473 (Norwegian School of Veterinary Science; Oslo, Norway), Umbelopsis isabellina UBOCC-A-101350 (Université de Bretagne Occidentale Culture Collection; Plouzané, France) and Penicillium glabrum FRR 4190 (Commonwealth Scientific and Industrial Research Organisation; North Ryde, Australia).

\section{Media and growth conditions}

Cultivation of fungi was first performed on agar media to obtain spores for the inoculation and then in nitrogen-limited liquid medium in order to stimulate lipid accumulation. For spore inoculum preparation the following agar media were used: malt extract agar (MEA) for M. circinelloides and P. glabrum and potato dextrose agar (PDA) for $U$. isabellina. MEA was prepared by dissolving $30 \mathrm{~g}$ malt extract (Merck, Germany), $5 \mathrm{~g}$ peptone (Amresco, USA) and $15 \mathrm{~g}$ agar powder (VWR Chemicals, Belgium) in $1 \mathrm{~L}$ distilled water and autoclaved at $115^{\circ} \mathrm{C}$ for $10 \mathrm{~min}$. PDA was prepared by dissolving $39 \mathrm{~g}$ potato dextrose agar (VWR Chemicals, Belgium) in $1 \mathrm{~L}$ distilled water and autoclaved at $121{ }^{\circ} \mathrm{C}$ for $15 \mathrm{~min}$. All agar cultivations were performed for 7 days at $25{ }^{\circ} \mathrm{C}$. Spores were harvested with a bacteriological loop from agar plates after the addition of $10 \mathrm{~mL}$ sterile physiologic salt solution. Spore concentrations were measured with hemocytometer (Fuchs-Rosenthal, Hausser Scientific Company, USA) and a DM6000B microscope (Leica Microsystems, Germany) and spore suspensions were diluted to $3.6 \times 10^{6}$ spore $\mathrm{mL}^{-1}$.

The broth medium was prepared according to the protocol described in Kavadia et al. [23] with modifications $\left(\mathrm{g} \mathrm{L}^{-1}\right)$ : glucose 80, yeast extract (Oxoid, England) 3, $\mathrm{KH}_{2} \mathrm{PO}_{4} 7, \mathrm{Na}_{2} \mathrm{HPO}_{4} 2, \mathrm{MgSO}_{4} \cdot 7 \mathrm{H}_{2} \mathrm{O} 1.5, \mathrm{CaCl}_{2} \cdot 2 \mathrm{H}_{2} \mathrm{O}$ $0.1, \mathrm{FeCl}_{3} \cdot 6 \mathrm{H}_{2} \mathrm{O} 0.008, \mathrm{ZnSO}_{4} \cdot 7 \mathrm{H}_{2} \mathrm{O} 0.001, \mathrm{CoSO}_{4} \cdot 7 \mathrm{H}_{2} \mathrm{O}$ $0.0001, \mathrm{CuSO}_{4} \cdot 5 \mathrm{H}_{2} \mathrm{O}$ 0.0001, $\mathrm{MnSO}_{4} \cdot 5 \mathrm{H}_{2} \mathrm{O} 0.0001$. Chemicals (except yeast extract) were purchased from Merck (Germany). Liquid medium was autoclaved for 
15 min at $121{ }^{\circ} \mathrm{C}$. The $\mathrm{pH}$ of the medium was 6.05 after sterilization. Cultivation in liquid medium was performed in the Duetz-MTPS (Enzyscreen, Netherlands), consisting of 24-square polypropylene deep well plates, low-evaporation sandwich covers and extra high cover clamps [24], which were mounted in two Innova 40R refrigerated desktop shakers (Eppendorf, Germany). Autoclaved and dried microtiter plates were filled with $2.5 \mathrm{~mL}$ of sterile liquid medium by using the Stepper 411 adjustable repeater pipette (Socorex, Switzerland). Each well was inoculated with $50 \mu \mathrm{L}$ fungal spore suspension. Cultivations were performed for 12 days at 20 and $30^{\circ} \mathrm{C}$ at $300 \mathrm{rpm}$ agitation speed (circular orbit $0.75^{\prime \prime}$ or $19 \mathrm{~mm}$ ). Each day, one plate was removed from both shakers for analysis.

\section{Experimental design}

All microplates were prepared in the following scheme: the first eight wells were inoculated with $M$. circinelloides, the second eight wells with $U$. isabellina and the last eight wells with $P$. glabrum. For each strain, fungal biomass of the first three wells, considered as biological replicates (210 samples in total), was used for lipid analysis by HTS-FTIR spectroscopy. Supernatant of the same wells, as well as the starting growth medium (215 samples in total), was used for glucose and protein analyses by HPLC and colorimetric assay. Finally, the merged biomass from the other five wells (70 samples in total) was used for lipid analysis by gas chromatography (GC).

\section{Bright-field and fluorescent microscopy}

Morphology of the filamentous fungi was examined with a DM6000B microscope (Leica Microsystems, Germany). Microscopic pictures were obtained with an Evolution MP camera kit (Media Cybernetics, USA). A Nile-red staining solution was prepared by dissolving $1 \mathrm{mg}$ Nilered crystals (Sigma-Aldrich, Germany) in $1 \mathrm{~mL}$ ethanol. Then, $10 \mu \mathrm{L}$ Nile-red solution was dried onto a glass side, the biomass was added and covered with a glass coverslip. Nile-red stained samples were incubated for $1 \mathrm{~h}$ at $4{ }^{\circ} \mathrm{C}$ in the dark and images were captured using a $490 \mathrm{~nm}$ excitation/530 nm emission wavelength filter cube (Leica Microsystems, Germany).

\section{Preparation of supernatant and biomass}

The supernatant was separated from the fungal biomass by transferring $2 \mathrm{~mL}$ fermentation broth with plastic Pasteur pipettes into Eppendorf tubes and the subsequent centrifugation at $13,000 \mathrm{rpm}$ for $20 \mathrm{~min}$ at $4{ }^{\circ} \mathrm{C}$. Fungal biomass from Eppendorf tubes were washed three times with cold distilled water and filtered under vacuum using a Whatman No. I filter paper (GE Whatman, USA). All samples were stored at $-20^{\circ} \mathrm{C}$ until analysis.

\section{Preparation of fungal biomass for FTIR analysis}

The washed fungal biomass (approx. $50 \mu \mathrm{L}$ per sample) was homogenized in 96-square deepwell plates with $500 \mu \mathrm{L}$ distilled water using a modular liquid handling robot [25] with integrated $2 \mathrm{~mm}$ single-pin Q55 sonicator (Qsonica, USA). The sonication was performed in a pulse regime with $15 \mathrm{~s}$ sonication time and $5 \mathrm{~s}$ washing time. Total sonication time for $U$. isabellina, $M$. circinelloides, and $P$. glabrum was $30 \mathrm{~s}, 1 \mathrm{~min}$ and $1.5 \mathrm{~min}$, respectively. P. glabrum biomass cultivated at $20{ }^{\circ} \mathrm{C}$ was manually sonicated for $2 \mathrm{~min}$, due to a rigid pellet structure, which was difficult to homogenize with the robotic system.

\section{FTIR spectroscopy}

FTIR analysis of the sonicated fungal biomass was performed using the High Throughput Screening eXTension (HTS-XT) unit coupled to the Vertex 70 FTIR spectrometer (both Bruker Optik, Germany) in transmission mode. From each suspension, $8 \mu \mathrm{L}$ were transferred to an IR-light-transparent silicon 384-well microplate (Bruker Optik, Germany) in three technical replicates. Samples were dried at room temperature for $2 \mathrm{~h}$ to form films that were suitable for FTIR analysis. The spectra were recorded in the region between 4000 and $500 \mathrm{~cm}^{-1}$ with a spectral resolution of $6 \mathrm{~cm}^{-1}$ and an aperture of $5.0 \mathrm{~mm}$. For each spectrum, 64 scans were averaged. Each spectrum was recorded as the ratio of the sample spectrum to the spectrum of the empty microplate. In total, 210 samples were measured and 630 FTIR spectra were obtained.

\section{Glucose analysis}

Glucose was quantified using an UltiMate 3000 UHPLC system (Thermo Scientific, USA) equipped with RFQFast Acid $\mathrm{H}+8 \%(100 \times 7.8 \mathrm{~mm})$ column (Phenomenex, USA) and coupled to a refractive index (RI) detector. Samples were diluted ten times before analysis, filter sterilized and were subsequently eluted isocratically at $0.6 \mathrm{~mL} \mathrm{~min}^{-1}$ flow rate in $12 \mathrm{~min}$ with $5 \mathrm{mM} \mathrm{H}_{2} \mathrm{SO}_{4}$ mobile phase at $85^{\circ} \mathrm{C}$ column temperature.

\section{Protein analysis}

Protein concentration in sample of supernatants was determined with a Bradford-method based colorimetric assay (Bio-Rad Protein Assay, USA) according to the microplate protocol. Absorbance was measured at $595 \mathrm{~nm}$ with a SPECTROstar Nano UV/Vis microplate reader (BMG Labtech, Germany). A calibration curve was prepared with media containing different amount of yeast extract. 


\section{Preparation of fungal biomass for FAME extraction and GC analysis}

The fermentation broth from the other five wells of the microplate were merged, filtered and washed as described above, frozen at $-20{ }^{\circ} \mathrm{C}$ and then lyophilized for 2 days in an Alpha 1-2 LDPlus freeze-dryer (Martin Christ, Germany) at $-55^{\circ} \mathrm{C}$ (condenser temperature) and 0.01 mbar pressure. The dried biomass was also used to calculate the cell dry weight (CDW). In order to obtain reliable dry cell weight data, the biomass grown on the walls of the wells was also collected and measured in contrast to that, used for FTIR and GC analysis.

\section{Lipid extraction}

Direct transesterification was performed according to Lewis et al. [26] with modifications for lipid extraction from fungal biomass: $2 \mathrm{~mL}$ screw-cap polypropylene (PP) tubes were filled, in three technical replicates, with $30 \pm 3 \mathrm{mg}$ freeze dried fungal biomass, $250 \pm 30 \mathrm{mg}$ (710-1180 $\mu \mathrm{m}$ diameter) acid-washed glass beads (Sigma-Aldrich, USA) and $600 \mu \mathrm{L}$ methanol. The fungal biomass was disrupted in a FastPrep-24 high-speed benchtop homogenizer (MP Biomedicals, USA) at $6.5 \mathrm{~m} \mathrm{~s}^{-1}$, for $1 \mathrm{~min}$ cycle length and 6 cycles. The disrupted fungal biomass was transferred into glass reaction tubes by washing the PP tube with $2400 \mu \mathrm{L}$ methanolchloroform-hydrochloric acid solvent mixture (7.6:1:1 $\mathrm{v} / \mathrm{v})$. Twenty microliters from a $25 \mathrm{mg} \mathrm{mL}^{-1}$ tridecanoic acid (C13:0, Sigma-Aldrich, USA) internal standard solution in methanol was added to the glass reaction tubes. The reaction mixture was vortexed for $10 \mathrm{~s}$ and incubated at $90{ }^{\circ} \mathrm{C}$ for $1 \mathrm{~h}$, followed by cooling to room temperature and addition of $1 \mathrm{~mL}$ distilled water. The fatty acid methyl esters (FAMEs) were extracted by the addition of $2 \mathrm{~mL}$ hexane-chloroform $(4: 1 \mathrm{v} / \mathrm{v})$ followed by $10 \mathrm{~s}$ vortex mixing. The reaction tubes were centrifuged at $3000 \mathrm{~g}$ for $10 \mathrm{~min}$ at $4{ }^{\circ} \mathrm{C}$ and the upper hexane phase was collected in glass tubes. The hexane-chloroform extraction was performed thrice. Subsequently, the solvent was evaporated under nitrogen at $60{ }^{\circ} \mathrm{C}$ and FAMEs were dissolved in $1.5 \mathrm{~mL}$ hexane containing $0.01 \%$ butylated hydroxytoluene (BHT, Sigma-Aldrich, USA). The extracted non-lipid cell compounds (insoluble in hexane) were removed after centrifugation in Eppendorf tubes at $15,000 \mathrm{~g}$ for $5 \mathrm{~min}$ at $4{ }^{\circ} \mathrm{C}$. The FAMEs dissolved in hexane were transferred to $\mathrm{GC}$ vials containing small amount of anhydrous sodium sulfate.

\section{GC fatty acid analysis}

Analysis of the extracted FAMEs was performed in a HP 6890 gas chromatograph (Hewlett Packard, USA) equipped with a SGE BPX70, $60.0 \mathrm{~m} \times 250 \mu \mathrm{m} \times 0.25 \mu \mathrm{m}$ column (SGE Analytical Science, Australia) and flame ionization detector (FID). Helium was used as a carrier gas. The runtime was $36.3 \mathrm{~min}$ with an initial oven temperature of $100{ }^{\circ} \mathrm{C}$, which was increased steadily to $220^{\circ} \mathrm{C}$ (4.3 min to $170{ }^{\circ} \mathrm{C}$, then $20 \mathrm{~min}$ to $200{ }^{\circ} \mathrm{C}$ and $12 \mathrm{~min}$ to $220{ }^{\circ} \mathrm{C}$ ). The injector temperature was $280{ }^{\circ} \mathrm{C}$ and $1 \mu \mathrm{L}$ was injected in split mode (50:1 split ratio). FAMEs were identified with a C4-C24 FAME standard mixture (18919-1AMP, Supelco, USA) dissolved in hexane, and were quantified by the C13:0 internal standard and relative response factors (RRF) calculated from 5-point calibration curves of the individual FAMEs in the standard mixture.

\section{Data analysis}

The FTIR spectra of fungal biomass were preprocessed in the following way: (1) technical replicates (630 spectra in total) were averaged resulting in 210 average spectra, (2) second derivative spectra were obtained by the Savitzky-Golay algorithm [27] using windows size 9 and a second degree polynomial, (3) Extended Multiplicative Signal Correction (EMSC), an MSC model extended by a linear and quadratic component, was employed on the combined spectral range comprising of 3100-2800 and of $1800-500 \mathrm{~cm}^{-1}$ spectral regions [28]. These spectral regions were selected since they contain bands distinctive for fungi $[18,29]$.

Principal component analysis (PCA) was applied in order to evaluate the differentiation ability of FTIR analysis between fungal species, cultivation temperature and time, and to compare sample variation pattern in FTIR and GC data concerning fatty acid composition. The preprocessed FTIR spectra (2nd derivative and EMSC) were used in the $3100-2800 \mathrm{~cm}^{-1}$ region, while the GC fatty acid data were autoscaled.

Partial least square regression (PLSR) was used to establish calibration models for fatty acid parameters of fungal biomass. In order to establish such models a data set of GC reference measurements (responses) were used as a $\mathrm{Y}$ matrix, which was regressed onto an $\mathrm{X}$ matrix containing FTIR measurements of fungal biomass (predictors). Fatty acid parameters included total lipid content of biomass and fatty acid compositional data such as saturated fatty acids (SAT), monounsaturated fatty acids (MUFA), polyunsaturated fatty acids (PUFA), unsaturation index and relevant single fatty acids. From a total of 210 averaged FTIR spectra, nine samples belonging to early growth phase of the fungi were excluded ( 3 biological replicates each of $M$. circinelloides $20^{\circ} \mathrm{C}$ on day 1 , P. glabrum $20{ }^{\circ} \mathrm{C}$ on day 2 , and P. glabrum $30{ }^{\circ} \mathrm{C}$ on day 1). The remaining 201 average FTIR spectra were used for the PLSR. Reference GC fatty acid dataset was built 
on the measurement of 67 fungal samples, each of them measured in three technical replicates and then results were averaged. Therefore, one averaged GC fatty acid composition was used in the regression for three corresponding biological replicate FTIR spectra. In order to optimize the number of PLSR principal components (PCs), cross-validation ( $\mathrm{CV}$ ) was performed, where cross validation segments were defined by days. All spectra from samples obtained on the same day were removed in turn and used for validating the model established on the rest of the data. A root-mean-square error (RMSE) was calculated for models with 1-25 components. The optimal model was the one with the lowest number of PCs having insignificantly higher RMSE than the model with the minimum RMSE.

The Unscrambler, V10.3 (CAMO, Norway) and Matlab, V8.5 (The Mathworks, USA) software were used to perform the data analysis.

\section{Results and discussion}

Growth and lipid accumulation of oleaginous fungi in Duetz-MTPS

The growth of dimorphic fungus $M$. circinelloides resulted in clumped mycelium mixed with dispersed yeast-like cells, while $U$. isabellina and P. glabrum grew in pellets of approx. 0.5-2 $\mathrm{mm}$ in diameter (Fig. 1). Maximum lipid bodies' diameter were 17, 5 and $2.5 \mu \mathrm{m}$ respectively (Fig. 1). It is worth mentioning that filling volume in the 24-well MTP should not exceed $2.5 \mathrm{~mL}$ at $300 \mathrm{rpm}$ agitation speed in order to avoid the contact of fermentation broth with sandwich cover, which potentially leads to cross-contamination.

For all three species, initial growth rate was higher at $30{ }^{\circ} \mathrm{C}$ than at $20{ }^{\circ} \mathrm{C}$ (Fig. 2a). Final biomass concentration (18-23 $\left.\mathrm{g} \mathrm{L}^{-1}\right)$ was similar for the three fungi at both temperatures. The exception was $M$. circinelloides grown at $20{ }^{\circ} \mathrm{C}$, which consumed less than half of the initial
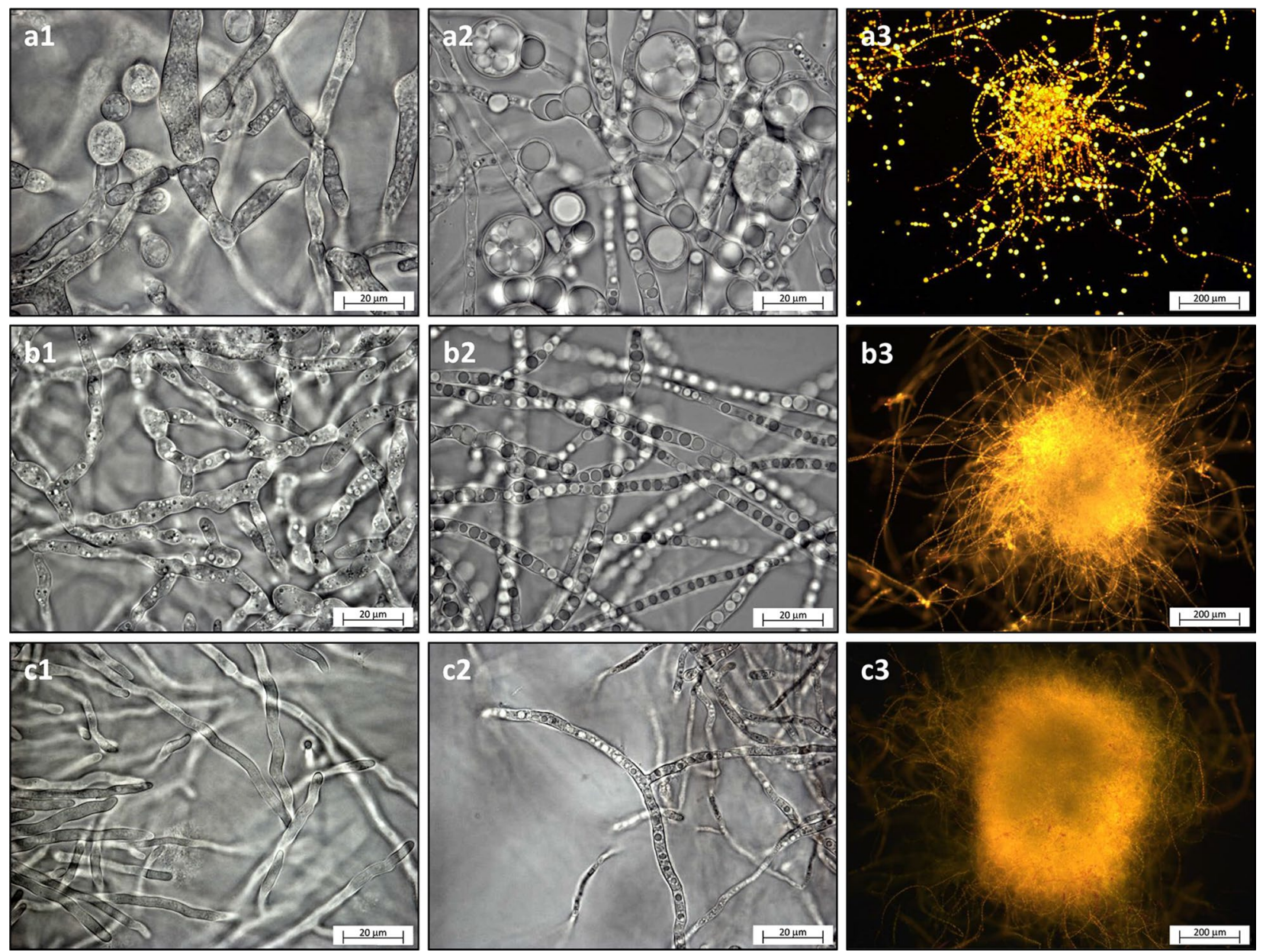

Fig. 1 Bright-field (a1-c2) and fluorescent microscopy (a3-c3) images of M. circinelloides (a), U. isabellina (b) and P. glabrum (c). Images (a1-c1) show fungal hyphae before or at the beginning of lipogenesis (day 1-2) while images (a2-c3) were captured from hyphae filled with distinct lipid bodies (day 3-6) 

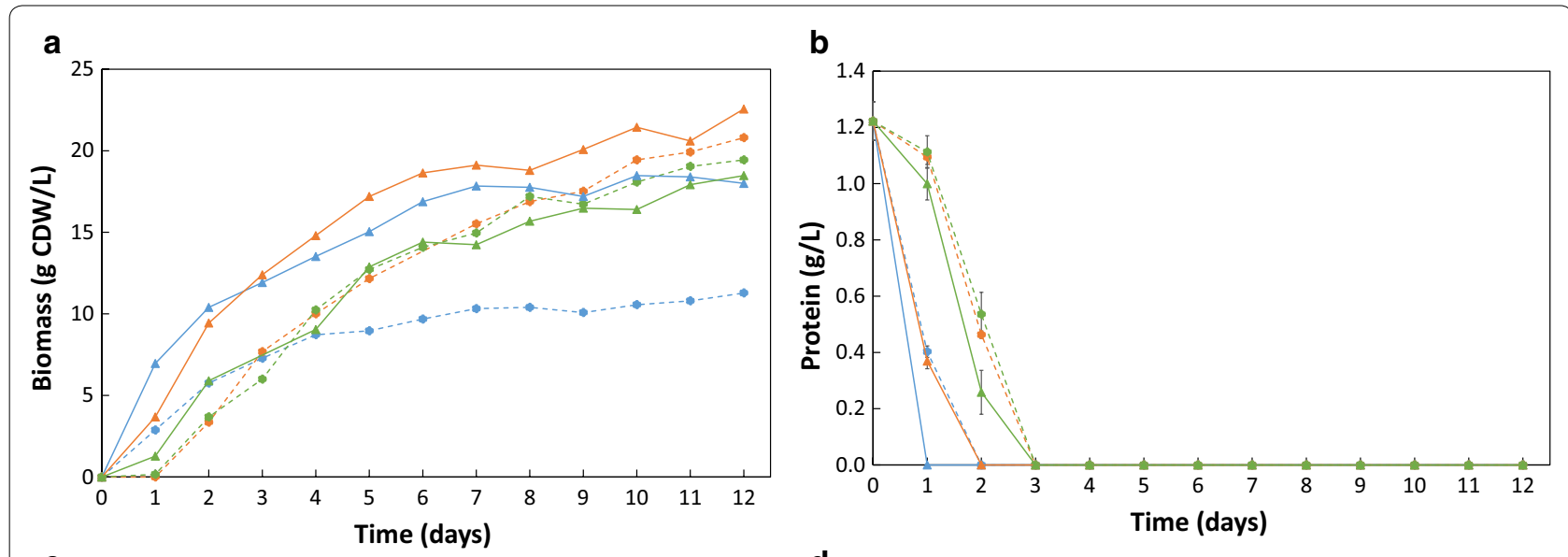

C

d
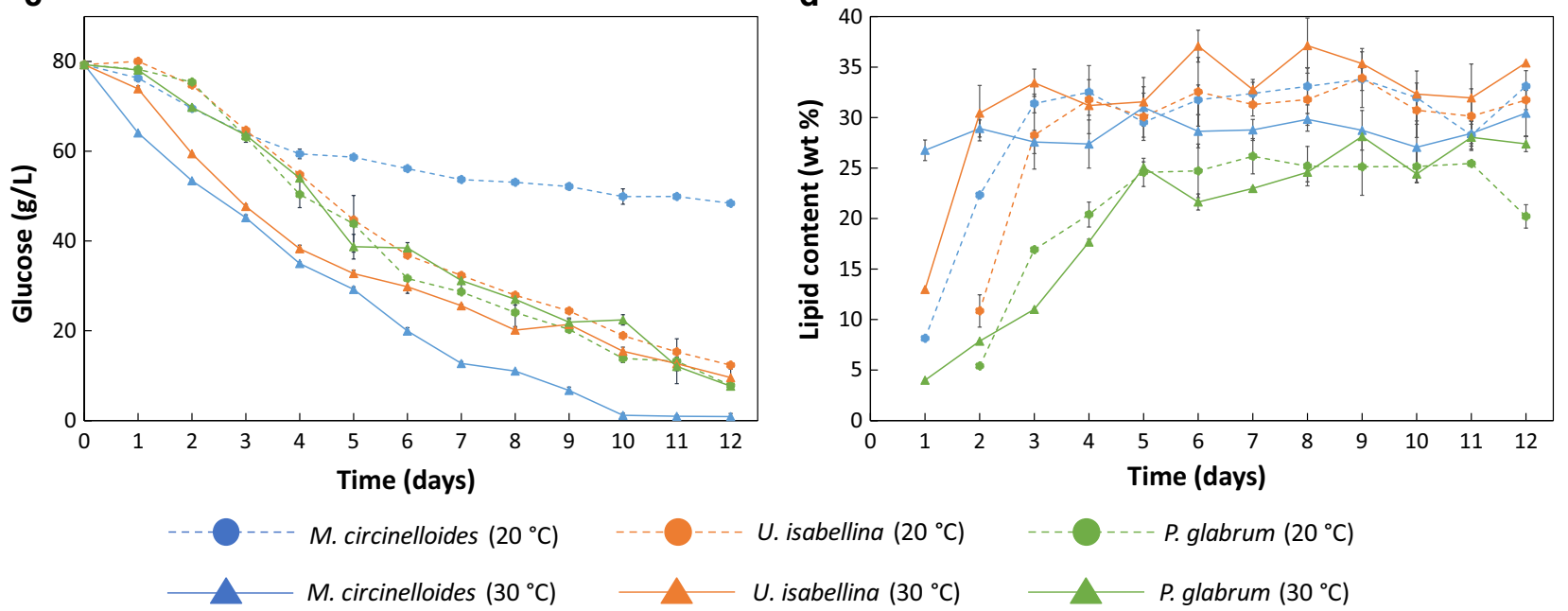

Fig. 2 Biomass (a), protein (b) and glucose (c) concentrations in cultivation medium and lipid content (d) of M. circinelloides, U. isabellina and $P$. glabrum cultivated at 20 and $30^{\circ} \mathrm{C}$ in the Duetz-MTPS $(n=3$, errorbars $=S D)$

glucose and reached only $11 \mathrm{~g} \mathrm{~L}^{-1}$ biomass. However, at $30{ }^{\circ} \mathrm{C}$, which is the optimum growth temperature for this species [30], M. circinelloides showed glucose depletion after 10 days of cultivation (Fig. 2c). Proteins from yeast extract were depleted within 1-3 days of cultivation for the three studied fungi (Fig. 2b), however growth continued at a reduced rate after nitrogen depletion. This result is in agreement with the study of Tang et al. [31], where the sole $\mathrm{N}$-source, i.e., ammonium tartrate was depleted by $M$. circinelloides within $9-10 \mathrm{~h}$, while growth continued until 60-80 h. M. circinelloides and $U$. isabellina reached $31-37 \%$ lipid content of the biomass, while $P$. glabrum accumulated 26-28\% lipid (Fig. 2d). Further fermentation results can be found in Additional file 1: Table S1 and Figure S1.

$M$. circinelloides and $U$. isabellina produced unsaturated fatty acids up to 18 carbon chain length gammalinolenic acid (C18:3n6 or GLA), while P. glabrum produced unsaturated fatty acids up to alpha-linolenic acid (C18:3n3 or ALA) (Fig. 3). These results are in agreement with previous studies, showing that more advanced fungi (Ascomycota and Basidiomycota) produce the $\mathrm{n}-3$ isomer of the $\mathrm{C} 18$ trienoic fatty acid, while basal fungi (Mucoromycotina, Chytridiomycota) produce the $\mathrm{n}-6$ isomer [32,33]. After 12 days cultivation, higher content of oleic acid (C18:1n9), GLA ( $M$. circinelloides and $U$. isabellina) and ALA (P. glabrum) was observed at $20^{\circ} \mathrm{C}$ than at $30^{\circ} \mathrm{C}$, while linoleic acid $(\mathrm{C} 18: 2 \mathrm{n} 6, \mathrm{LA})$ content was higher at $30^{\circ} \mathrm{C}$ than at $20^{\circ} \mathrm{C}$ for all the three studied fungi. The PUFA content in the oil, including LA, GLA and ALA rapidly decreased after the transition from exponential growth to stationary phase and remained relatively stable afterwards. In the stationary phase of $M$. circinelloides and $U$. isabellina, oleic acid content tended to increase concomitant with decrease in saturated fatty acids (C16:0, palmitic acid and C18:0, stearic acid) (Additional file 1: Figure S2). 


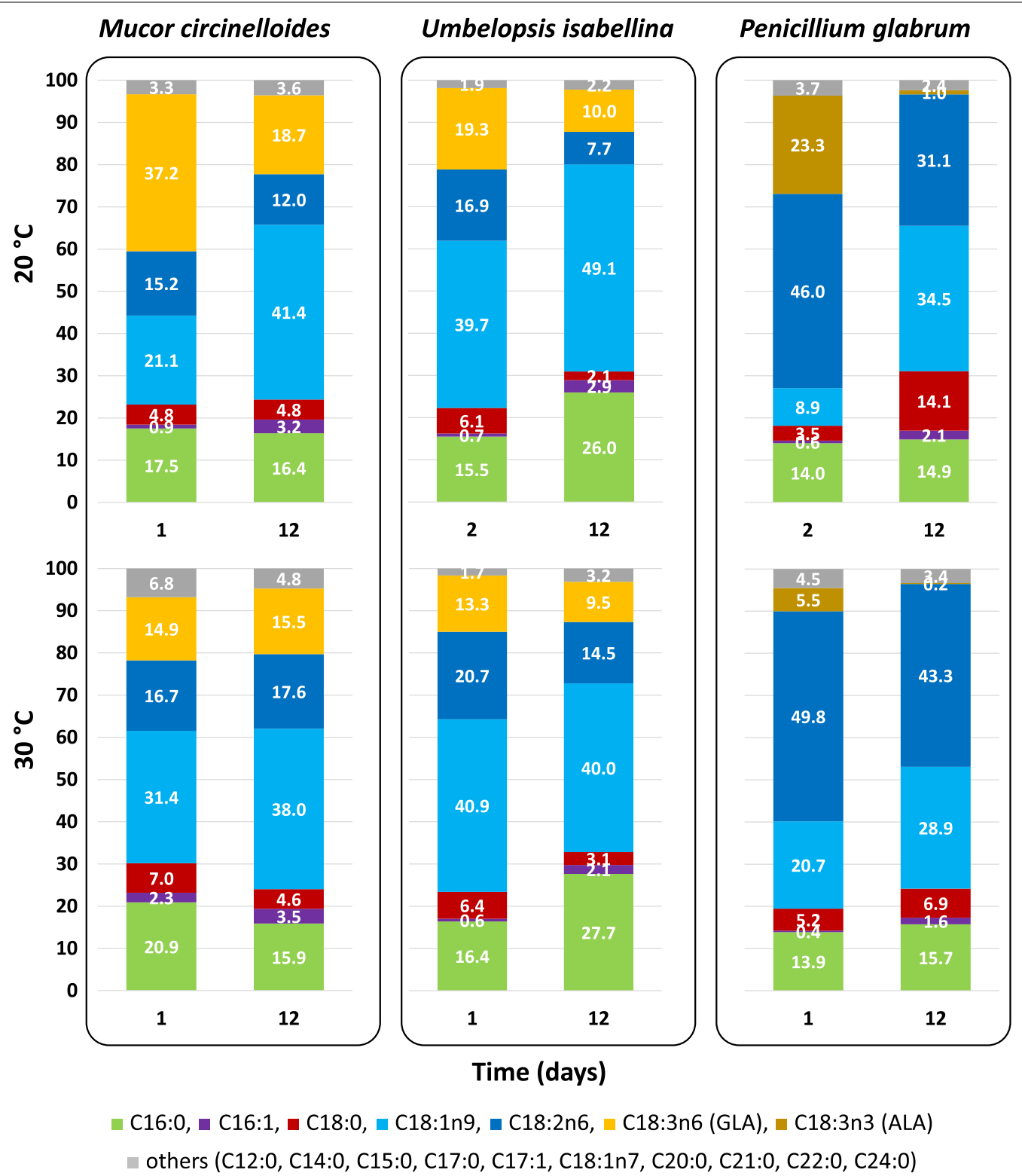

Fig. 3 Fatty acid composition (\%) of M. circinelloides, U. isabellina and P. glabrum cultivated at $20^{\circ} \mathrm{C}$ and at $30^{\circ} \mathrm{C}$ at first and last day of sampling in the Duetz-MTPS. Values represent mean value of three extraction - GC replicate measurements. Coefficient of variation was less than $2 \%$ for main fatty acids

FTIR spectroscopy of oleaginous filamentous fungi

The FTIR spectra of $M$. circinelloides, $U$. isabellina and P. glabrum biomass after 1 and 12 days of cultivation are shown in Fig. 4. Assigned spectral bands of the FTIR spectrum and their respective functional groups are listed in Additional file 1: Table S2. In the infrared spectrum, cellular lipids were represented by several peaks, related to different lipid functional groups: (I) peaks in the regions $3050-2800 \mathrm{~cm}^{-1}$ (peaks $\mathrm{N}^{\circ} 1-4$ in Fig. 4), $1500-1300 \mathrm{~cm}^{-1}$ (No $\left.8-10\right)$ and at $725 \mathrm{~cm}^{-1}\left(\mathrm{~N}^{\circ} 16\right)$ are related to lipid acyl chains, (II) peak around $3008 \mathrm{~cm}^{-1}$ $\left(\mathrm{N}^{\circ} 1\right)$ corresponds to $=\mathrm{C}-\mathrm{H}$ stretching and gives indication about the lipid unsaturation index [14, 15, 22], (III) the peak at $1745 \mathrm{~cm}^{-1}\left(\mathrm{~N}^{\circ} 5\right)$ is related to the ester carbonyl bond, which represents the vast majority of the total lipid content in the cell $[14,22]$. We observed a relative increase with the cultivation time for all lipid related bands in mid-IR spectra of all studied fungi, indicating that during cultivation in nitrogen limited medium, the main biochemical changes in fungi were related to 


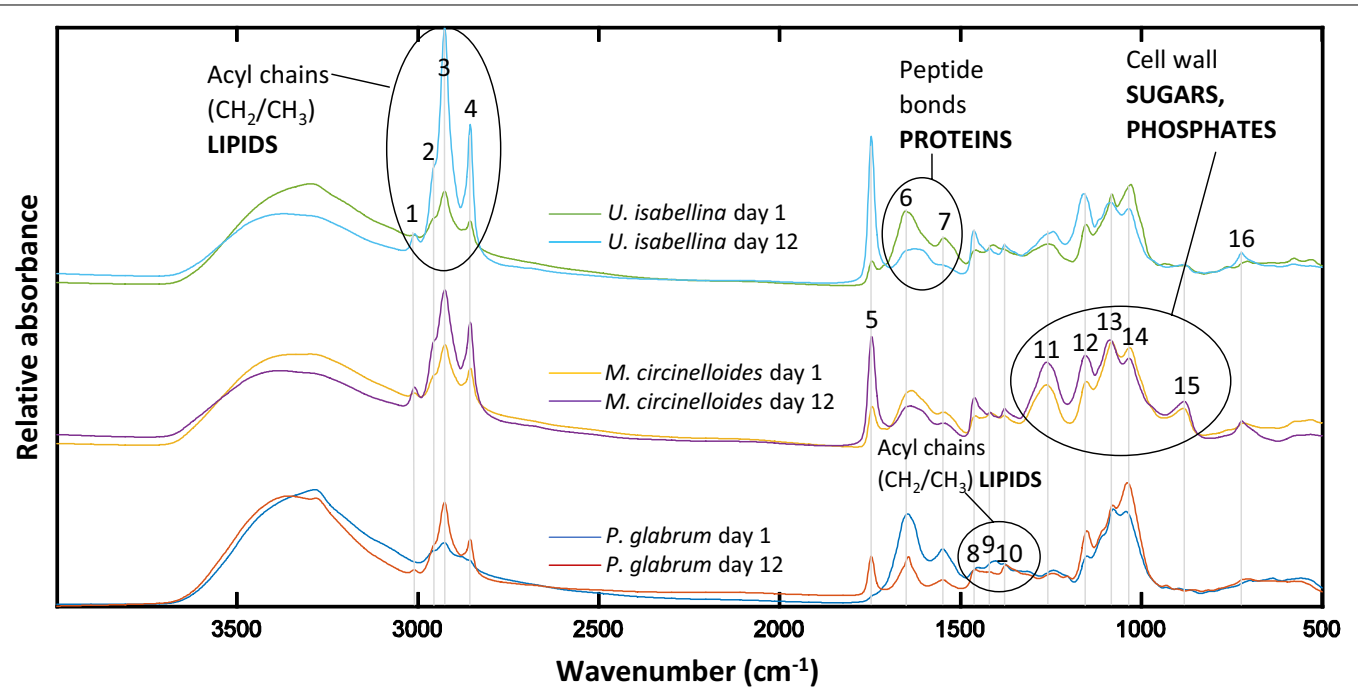

Fig. 4 FTIR spectra of P. glabrum, M. circinelloides, U. isabellina $\left(30^{\circ} \mathrm{C}\right)$ after 1 and 12 days of cultivation

intracellular lipid accumulation. As a consequence, the relative absorbance of protein-related peaks between 1690 and $1500 \mathrm{~cm}^{-1}$, which are mainly influenced by the amide I (peak $\mathrm{N}^{\circ} 6$ in Fig. 4) and amide II bands ( $\mathrm{N}^{\circ} 7$ ) [8], decreased during lipid accumulation.

Temporal-dependent changes in complex polysaccharide region $\left(1240-900 \mathrm{~cm}^{-1}\right)$ related to phosphate groups (nucleic acids, polyphosphates, phospholipids), as well as $\mathrm{C}-\mathrm{O}$ and $\mathrm{C}-\mathrm{O}-\mathrm{C}$ absorption peaks of the cell wall polysaccharides (chitin, chitosan, $\beta$-glucan, mannan, etc.), were also observed for all fungi. The appearance of the strong peaks around 1260 (peak No 11 in Fig. 4) and $880 \mathrm{~cm}^{-1}$ ( $\left.\mathrm{N}^{\circ} 15\right)$ in $M$. circinelloides spectra indicated an increase in polyphosphate content in its cell wall, as reported previously [34]. The $U$. isabellina IR spectra showed an absorbance increase at $1240-1250 \mathrm{~cm}^{-1}$, that may also indicate a polyphosphate content increase, but this increase was less prominent than for $M$. circinelloides. Relative to amide I peak, the $\beta(1,3)$-glucan peaks (at 1150 and $1080 \mathrm{~cm}^{-1}$ ) (peaks $\mathrm{N}^{\circ} 12$ and 13 in Fig. 4) and the $\mathrm{C}-\mathrm{O}$ stretching peak around $1033 \mathrm{~cm}^{-1}$ (No 14) increased as well with the cultivation time for all studied fungi. Such changes in the polysaccharide region of IR spectra show that the cell wall composition changes during lipid accumulation. We can hypothesize that an increase of $\beta(1,3)$-glucan peaks corresponds to an increase in the cell wall thickness which goes along with lipid accumulation. Indeed, the homogenization of lipid-rich fungal biomass used for FTIR required long time, while that used for $\mathrm{GC}$ analysis required the use of hydrochloric acid and harsh mechanical pretreatment (bead beating) [34]. Ami et al. [8] and Signori et al. [10] observed similar cell wall changes in FTIR spectra during oleaginous yeast lipogenesis.

PCA was performed on FTIR spectra and GC fatty acid data to evaluate temporal-, species- and cultivation temperature specific differences related to lipid production. The score plot of the first and second component of PCA of the FTIR data shows clustering according to the fungal species and cultivation temperatures (Fig. 5). The sample variation pattern in GC (Fig. 5a) and FTIR (Fig. 5b) score plots show similar tendencies and demonstrate that the main changes in fatty acid composition occur during the transition from growth phase (day 1-3) to the lipid accumulation phase (day 3-12). After 3 days of fermentation the fatty acid composition stabilizes. Furthermore, both GC and FTIR score plots indicate that reaching the stable fatty acid composition took longer for P. glabrum than for $M$. circinelloides and $U$. isabellina due to lower growth rate of this species. Biological replicate samples, which are marked by the same names and colors in the FTIR score plot, are close to each other. Variability between technical and biological replicates of the FTIR measurements were quantified by Pearson correlation coefficient (Additional file 1: Table S3). Technical and biological sample variability were of the same order, and two orders lower than variation between samples during the 12 days of cultivation. This demonstrates that the Duetz-system is a suitable high-throughput platform for the reproducible cultivation of filamentous fungi and that HTS-FTIR spectroscopy is a suitable high-throughput platform for the reproducible monitoring of lipogenesis of oleaginous fungi. 
a
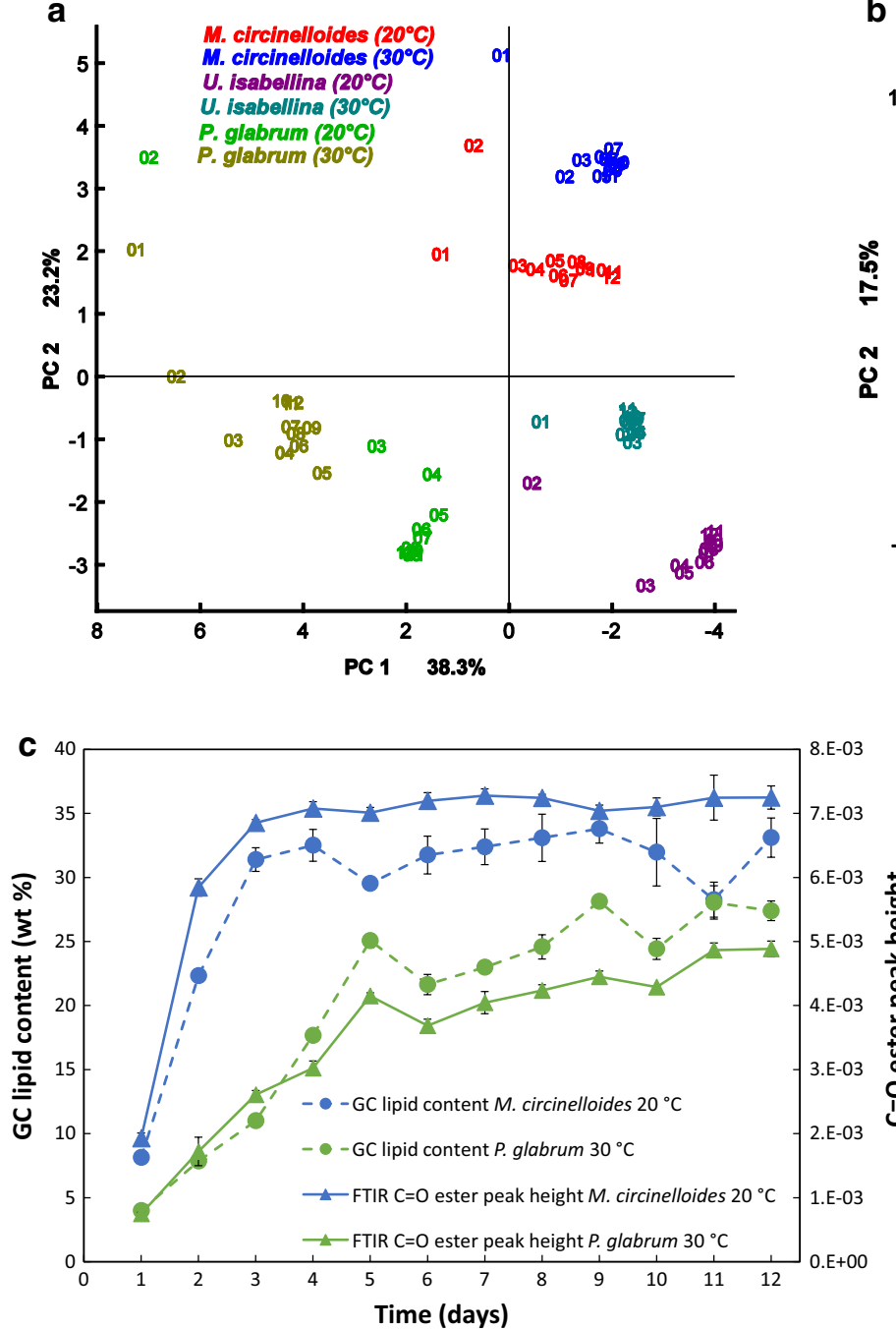
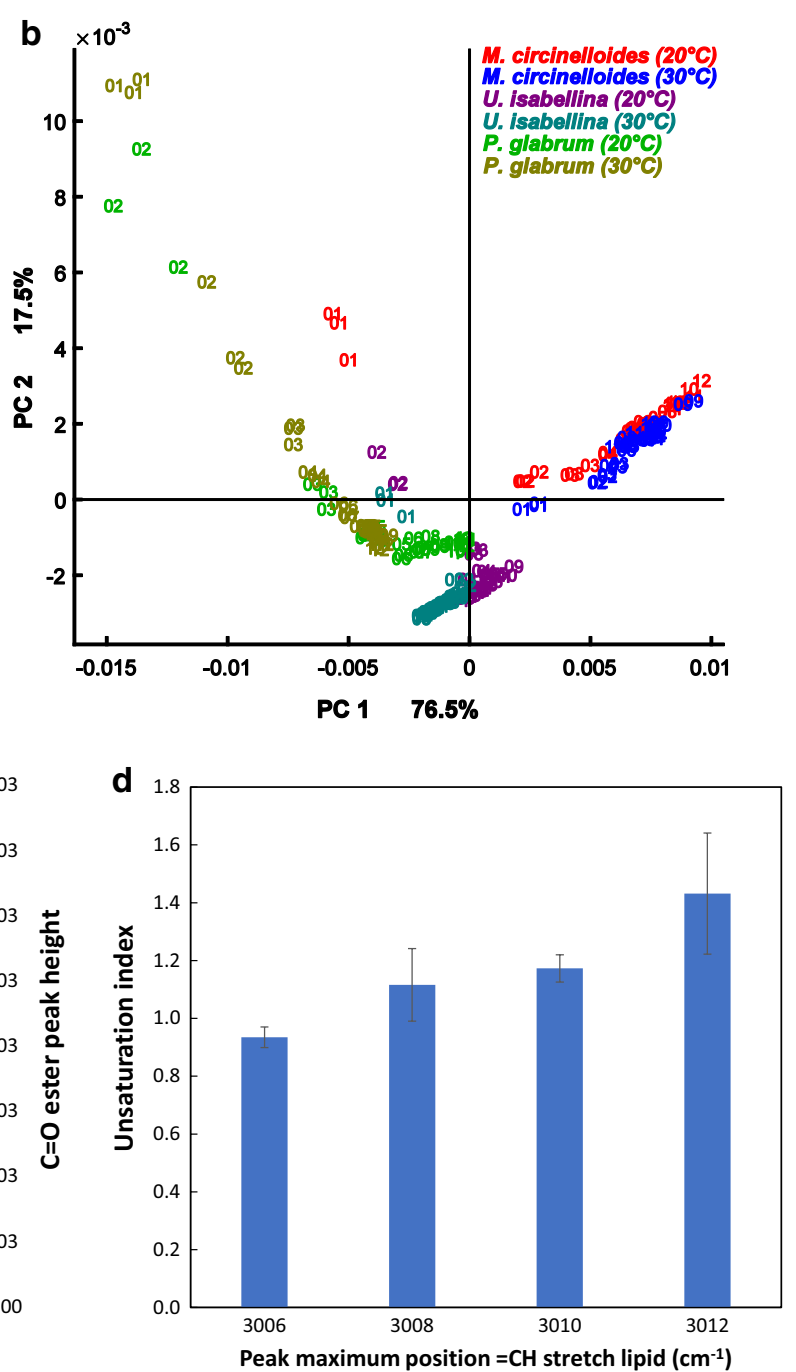

Fig. 5 Exploratory analysis of FTIR data and comparison with GC reference data. a First and second scores (PC1, PC2) in PCA of the auto-scaled GC fatty acid data (PC1 axis is reversed in the GC score plot). $\mathbf{b}$ First and second scores in PCA of the preprocessed FTIR spectra in the spectral range of $3100-2800 \mathrm{~cm}^{-1}$. c Total lipid content measured by reference GC method and followed by the $C=0$ ester peak height from the pre-processed FTIR spectra $(n=3$, error bars $=\mathrm{SD})$. $\mathbf{d}$ Relationship between unsaturation indices and position of the $=\mathrm{C}-\mathrm{H}$ stretching bond peak maxima $\left(\mathrm{cm}^{-1}\right)$ in FTIR spectra. Unsaturation index $=[\Sigma(\%$ monoene $+2 \times \%$ diene $+3 \times \%$ triene $)] / 100[35]$

The total lipid content of the biomass was monitored using the $\mathrm{C}=\mathrm{O}$ ester peak height $\left(1745 \mathrm{~cm}^{-1}\right)$ of the preprocessed FTIR spectra. Total lipid trends for fungal biomass were assessed by the $\mathrm{C}=\mathrm{O}$ ester peak height and compared to reference GC total lipid measurement (Additional file 1: Figure S3). For example, Fig. 5c shows that the GC and FTIR based lipid content of biomass curves for M. circinelloides $\left(20^{\circ} \mathrm{C}\right)$ and P. glabrum $\left(30^{\circ} \mathrm{C}\right)$ are well correlated. The fluctuation observed in the GC total lipid data may be due to the extraction-transesterification protocol for GC measurement, which is more prone to measurement errors than the FTIR measurement, which is done on almost intact cells. Although FTIR total lipid data cannot be used for absolute quantification without calibration to reference analysis, it provides a reliable and rapid qualitative method to monitor lipid accumulation during the cultivation of oleaginous species. 
The position of the olefinic group around $3010 \mathrm{~cm}^{-1}$ is known to be related to the degree of unsaturation of fatty acids. It has been shown that a shift toward higher wavenumber suggests higher degree of unsaturation [14, 15]. In this study the peak position at $3006 \mathrm{~cm}^{-1}$ corresponded to an unsaturation index of $0.93 \pm 0.04$, while peak position $3012 \mathrm{~cm}^{-1}$ was described by an unsaturation index of $1.43 \pm 0.21$. Peak maxima between 3008 and $3010 \mathrm{~cm}^{-1}$ corresponded to an unsaturation index of $0.99-1.22$ (Fig. 5d).

For the quantitative prediction of fatty acid composition and total lipid content of biomass PLSR models were established by using FTIR spectra (Additional file 2: Table S5) as $\mathrm{X}$ variables (or predictors) and GC fatty acid data (Additional file 3: Table S6) as Y variables (or responses). For seven of the fatty acid parameters, excellent calibration results were achieved with regression coefficients $R^{2}$ above 0.91 and for three fatty acid parameters, good models were obtained with $\mathrm{R}^{2}$ between 0.86 and 0.89 (Table 1). Residual predictive deviation of cross-validation $\left(\mathrm{RPD}_{\mathrm{CV}}\right)$ values were good to acceptable between 2.6 and 5.1. The slightly lower prediction ability for total lipid content might be due to the high variability in GC quantification (Fig. 2d). The models for MUFA and oleic acid (C18:1n9) had a high complexity involving a higher number of PLS factors compared to the other models. This high complexity may involve some degree of over-fitting. The predictions of linolenic acid and unsaturation index are shown in Additional file 1: Figure S4, where the predicted values are plotted against the measured values for both prediction and validation results. Since both prediction and validation results show the same high prediction ability, we can conclude that the calibration models are stable. Prediction result including all 210 samples can be found in Additional file 1: Table S4.

\section{Conclusions}

In this study, we have examined a high-throughput approach for the cultivation and monitoring of oleaginous fungi. For this purpose, a Duetz microtiter plate system was combined with rapid FTIR spectroscopy of fungal biomass. First, the microcultivation performance was evaluated for the studied fungi. Biological replicate cultivations in the 24 well deep well microtiter plate showed excellent reproducibility based on glucose consumption (pooled standard deviation $=1.1 \mathrm{~g} \mathrm{~L}^{-1}$ glucose) and FTIR spectra of biomass (average Pearson correlation coefficient $=0.9994$ ). Fungal cultures with high biomass concentrations (up to $23 \mathrm{~g} \mathrm{~L}^{-1} \mathrm{CDW}$ ) and high lipid content (up to 35\%) were achieved in the Duetz-MTPS.

Evaluation of the FTIR spectra of fungi during fermentation resulted in lipid accumulation curves that followed very similar trends to total lipid curves obtained by reference GC quantification. In general, the lipid accumulation curves on the basis of the FTIR $\mathrm{C}=\mathrm{O}$ ester peak showed smaller day-to-day variations and thus are more plausible. For quantitative purposes, the FTIR spectral data of biomass were calibrated versus GC fatty acid data. Summed fatty acid parameters (SAT, MUFA, PUFA, total fat, unsaturation index) and main individual fatty acids were predicted with high precision $\left(R^{2}\right.$ between 0.86 and $0.96, \mathrm{RPD}_{\mathrm{CV}}$ between 2.6 and 5.1). Sample preparation for HTS-FTIR measurement can be fully automated by robotics to further increase precision and throughput. We therefore conclude that HTS-FTIR spectroscopy is a simple, rapid tool for screening or at-line monitoring the cultivation of oleaginous species.

Table 1 PLS regression results between HTS-FTIR and GC fatty acid measurements $(\mathbf{N}=201)$

\begin{tabular}{|c|c|c|c|c|c|c|c|}
\hline Fatty acid & Range & Mean & $\begin{array}{l}\text { Standard } \\
\text { deviation }\end{array}$ & $\mathrm{R}^{2 \mathrm{a}}$ & RMSECV $^{\mathbf{b}}$ & $\operatorname{RPD}_{\mathrm{CV}}^{\mathrm{c}}$ & PLS factors \\
\hline C16:0 & $13.4-31.9$ & 20.2 & 6.1 & 0.94 & 1.5 & 4.0 & 6 \\
\hline C18:0 & $2.1-14.4$ & 6.3 & 3.2 & 0.94 & 0.8 & 4.2 & 6 \\
\hline C18:1n9 & $25.4-49.1$ & 37.4 & 5.4 & 0.89 & 1.8 & 3.0 & 21 \\
\hline C18:2n6 & $7.6-48.1$ & 20.8 & 11.5 & 0.96 & 2.3 & 5.0 & 7 \\
\hline C18:3n6 & $0.0-22.3$ & 9.1 & 7.2 & 0.96 & 1.4 & 5.1 & 3 \\
\hline SAT & $22.4-39.2$ & 29.0 & 4.5 & 0.87 & 1.6 & 2.8 & 6 \\
\hline MUFA & $27.1-52.6$ & 40.7 & 5.6 & 0.93 & 1.5 & 3.9 & 21 \\
\hline PUFA & $15.9-50.5$ & 30.3 & 8.1 & 0.93 & 2.2 & 3.7 & 7 \\
\hline Unsaturation index & $0.89-1.33$ & 1.11 & 0.13 & 0.95 & 0.03 & 4.5 & 9 \\
\hline Total lipid & $7.9-37.1$ & 27.8 & 6.1 & 0.86 & 2.3 & 2.6 & 4 \\
\hline
\end{tabular}

\footnotetext{
$\mathrm{R}^{2}$, cross-validated squared correlation coefficient

b RMSECV, root mean square error of cross validation

${ }^{c} \mathrm{RPD}_{\mathrm{CV}}$, residual predictive deviation of cross-validation (standard deviation/RMSECV)
} 


\section{Additional files}

Additional file 1. Figures S1-S4, Tables S1-S4. Additional figures and tables.

Additional file 2: Table S5. HTS-FTIR spectra of biomass.

Additional file 3: Table S6. GC fatty acid composition.

Additional file 4: Table S7. HPLC glucose concentration.

\section{Abbreviations}

Duetz-MTPS: Duetz microtiter plate system; EMSC: extended multiplicative signal correction; HTS: high-throughput screening; FTIR: Fourier transform infrared spectroscopy; MUFA: monounsaturated fatty acids; SAT: saturated fatty acids; PUFA: polyunsaturated fatty acids; PCA: Principal Component Analysis; PLSR: partial least squares regression.

\section{Authors' contributions}

Conceived the research idea: AK, VS. Designed the experiments: GK, AK, BZ, VS. Methodology: GK, VT, BZ, KF, VS. Performed the experiments: GK. Discussed the results: GK, AK, BZ, VT, VS, NKA. Analyzed the data: GK, VT, BZ, VS. Wrote the manuscript: GK. Discussed and revised the manuscript: GK, AK, VT, BZ, KF, DM, NKA, KV, SJH, JM, VS. All authors read and approved the final manuscript.

\section{Author details}

${ }^{1}$ Faculty of Science and Technology, Norwegian University of Life Sciences, Postbox 5003, 1432 Ås, Norway. ${ }^{2}$ Nofima AS, Osloveien 1, 1430 Ås, Norway.

${ }^{3}$ Faculty of Chemistry, Biotechnology and Food Science, Norwegian University of Life Sciences, Postbox 5003, 1432 Ås, Norway. ${ }^{4}$ Université de Brest, EA3882 Laboratoire Universitaire de Biodiversité et Ecologie Microbienne, IBSAM, ESIAB, Technopôle Brest Iroise, 29280 Plouzané, France.

\section{Acknowledgements}

The authors would like to acknowledge Sandeep Sharma and Elin Merete Wetterhus for their help with HPLC and GC measurements respectively, and Murat Bagcioglu for his help in data analysis. Wouter Duetz is also acknowledged for his valuable technical advices in microtiter plate cultivation.

\section{Competing interests}

The authors declare that they have no competing interests.

\section{Availability of data and materials}

All data generated or analysed during this study are included in this published article (and its additional files).

\section{Funding}

This work was supported by the Norwegian Research Council-BIONÆR Grant project number: 234258/E50 and the Norwegian Research Council "Interest" Grant, project number 227356.

\section{Publisher's Note}

Springer Nature remains neutral with regard to jurisdictional claims in published maps and institutional affiliations.

Received: 24 January 2017 Accepted: 5 June 2017 Published online: 09 June 2017

\section{References}

1. Ratledge C. Fatty acid biosynthesis in microorganisms being used for single cell oil production. Biochimie. 2004;86:807-15.

2. Ward OP, Singh A. Omega-3/6 fatty acids: alternative sources of production. Process Biochem. 2005;40:3627-52.

3. Linde T, Hansen NB, Lübeck M, Lübeck PS. Fermentation in 24-well plates is an efficient screening platform for filamentous fungi. Lett Appl Microbiol. 2014;59:224-30.
4. Long Q, Liu X, Yang Y, Li L, Harvey L, McNeil B, Bai Z. The development and application of high throughput cultivation technology in bioprocess development. J Biotechnol. 2014;192:323-38.

5. Meyer $V$, Andersen MR, Brakhage AA, et al. Current challenges of research on filamentous fungi in relation to human welfare and a sustainable bioeconomy: a white paper. Fungal Biol Biotechnol. 2016;3:6.

6. Rumin J, Bonnefond H, Saint-Jean B, Rouxel C, Sciandra A, Bernard O, Cadoret JP, Bougaran G. The use of fluorescent Nile red and BODIPY for lipid measurement in microalgae. Biotechnol Biofuels. 2015:8:42.

7. Meng Y, Yao C, Xue S, Yang H. Application of Fourier transform infrared (FT-IR) spectroscopy in determination of microalgal compositions. Bioresour Technol. 2014;151:347-54.

8. Ami D, Posteri R, Mereghetti P, Porro D, Doglia SM, Branduardi P. Fourier transform infrared spectroscopy as a method to study lipid accumulation in oleaginous yeasts. Biotechnol Biofuels. 2014;7:12.

9. Münchberg U, Wagner L, Spielberg ET, Voigt K, Rösch P, Popp J. Spatially resolved investigation of the oil composition in single intact hyphae of Mortierella spp. with micro-Raman spectroscopy. Biochim Biophys Acta Mol Cell Biol Lipids. 2013;1831:341-9.

10. Signori L, Ami D, Posteri R, Giuzzi A, Mereghetti P, Porro D, Branduardi P. Assessing an effective feeding strategy to optimize crude glycerol utilization as sustainable carbon source for lipid accumulation in oleaginous yeasts. Microb Cell Fact. 2016;15:75.

11. Kohler A, Bocker U, Shapaval V, Forsmark A, Andersson M, Warringer J, Martens H, Omholt SW, Blomberg A. High-throughput biochemical fingerprinting of Saccharomyces cerevisiae by Fourier transform infrared spectroscopy. PLoS ONE. 2015;10:e0118052.

12. Dean AP, Sigee DC, Estrada B, Pittman JK. Using FTIR spectroscopy for rapid determination of lipid accumulation in response to nitrogen limitation in freshwater microalgae. Bioresour Technol. 2010;101:4499-507.

13. Jungandreas $A$, Costa BS, Jakob T, Von Bergen M, Baumann S, Wilhelm C The acclimation of Phaeodactylum tricornutum to blue and red light does not influence the photosynthetic light reaction but strongly disturbs the carbon allocation pattern. PLoS ONE. 2014;9:e99727.

14. Vongsvivut J, Heraud P, Gupta A, Thyagarajan T, Puri M, McNaughton D, Barrow CJ. Synchrotron-FTIR microspectroscopy enables the distinction of lipid accumulation in thraustochytrid strains through analysis of individual live cells. Protist. 2015;166:106-21.

15. Shapaval V, Afseth NK, Vogt G, Kohler A. Fourier transform infrared spectroscopy for the prediction of fatty acid profiles in Mucor fungi grown in media with different carbon sources. Microb Cell Fact. 2014;13:86.

16. Janbu AO, Møretrø T, Bertrand D, Kohler A. FT-IR microspectroscopy: a promising method for the rapid identification of Listeria species. FEMS Microbiol Lett. 2008;278:164-70.

17. Santos C, Fraga ME, Kozakiewicz Z, Lima N. Fourier transform infrared as a powerful technique for the identification and characterization of filamentous fungi and yeasts. Res Microbiol. 2010;161:168-75.

18. Shapaval V, Schmitt J, Møretrø T, Suso H, Skaar I, Åsli A, Lillehaug D, Kohler A. Characterization of food spoilage fungi by FTIR spectroscopy. J Appl Microbiol. 2013;114:788-96.

19. Beekes M, Lasch P, Naumann D. Analytical applications of Fourier transform-infrared (FT-IR) spectroscopy in microbiology and prion research. Vet Microbiol. 2007;123:305-19.

20. Zimmermann B, Bagcioglu M, Sandt C, Kohler A. Vibrational microspectroscopy enables chemical characterization of single pollen grains as well as comparative analysis of plant species based on pollen ultrastructure. Planta. 2015;242:1237-50.

21. Veale EL, Irudayaraj J, Demirci A. An on-line approach to monitor ethanol fermentation using FTIR spectroscopy. Biotechnol Prog. 2007:23:494-500.

22. Ami D, Mereghetti P, Doglia SM. Multivariate analysis for Fourier transform infrared spectra of complex biological systems and processes. In: Valim de Freitas L, Barbosa Rodrigues de Freitas AP, editors. Multivariate analysis in management, engineering and the sciences. InTech; 2013. doi:10.5772/53850.

23. Kavadia A, Komaitis M, Chevalot I, Blanchard F, Marc I, Aggelis G. Lipid and gamma-linolenic acid accumulation in strains of zygomycetes growing on glucose. J Am Oil Chem Soc. 2001;78:341-6.

24. Duetz WA, Ruedi L, Hermann R, O'Connor K, Buchs J, Witholt B. Methods for intense aeration, growth, storage, and replication of bacterial strains in microtiter plates. Appl Environ Microbiol. 2000;66:2641-6. 
25. Li J, Shapaval V, Kohler A, Talintyre R, Schmitt J, Stone R, Gallant AJ, Zeze DA. A modular liquid sample handling robot for high-throughput Fourier transform infrared spectroscopy. In: Ding X, Kong X, Dai SJ, editors. Advances in reconfigurable mechanisms and robots II. Cham: Springer International Publishing; 2016. p. 769-78.

26. Lewis T, Nichols PD, McMeekin TA. Evaluation of extraction methods for recovery of fatty acids from lipid-producing microheterotrophs. J Microbiol Methods. 2000;43:107-16.

27. Savitzky A, Golay MJE. Smoothing + differentiation of data by simplified least squares procedures. Anal Chem. 1964;36:1627.

28. Kohler A, Kirschner C, Oust A, Martens H. Extended multiplicative signal correction as a tool for separation and characterization of physical and chemical information in Fourier transform infrared microscopy images of cryo-sections of beef loin. Appl Spectrosc. 2005;59:707-16.

29. Shapaval V, Møretrø T, Suso HP, Åsli AW, Schmitt J, Lillehaug D, Martens H, Böcker U, Kohler A. A high-throughput microcultivation protocol for FTIR spectroscopic characterization and identification of fungi. J Biophotonics. 2010;3:512-21.
30. Michailides TJ. Characterization and comparative-studies of Mucor isolates from stone fruits from California and Chile. Plant Dis. 1991;75:373-80.

31. Tang X, Chen HQ, Chen YQ, Chen W, Garre V, Song YD, Ratledge C. Comparison of biochemical activities between high and low lipid-producing strains of Mucor circinelloides: an explanation for the high oleaginicity of strain WJ11. PloS ONE. 2015;10:e0128396.

32. Broughton R. Omega 3 fatty acids: identification of novel fungal and chromistal sources. London: University of London, Royal Holloway; 2012.

33. Weete JD, Shewmaker F, Gandhi SR. gamma-Linolenic acid in zygomycetous fungi: syzygites megalocarpus. J Am Oil Chem Soc. 1998;75:1367-72.

34. Forfang K, Zimmermann B, Kosa G, Kohler A, Shapaval V. FTIR spectroscopy for evaluation and monitoring of lipid extraction efficiency for oleaginous fungi. PLoS ONE. 2017;12:e0170611.

35. Suutari M. Effect of growth temperature on lipid fatty acids of four fungi (Aspergillus niger, Neurospora crassa, Penicillium chrysogenum, and Trichoderma reesei). Arch Microbiol. 1995;164:212-6.

\section{Submit your next manuscript to BioMed Central and we will help you at every step:}

- We accept pre-submission inquiries

- Our selector tool helps you to find the most relevant journal

- We provide round the clock customer support

- Convenient online submission

- Thorough peer review

- Inclusion in PubMed and all major indexing services

- Maximum visibility for your research

Submit your manuscript at www.biomedcentral.com/submit 